Unexpected systematic degeneracy in a system of two coupled Gaudin models with homogeneous couplings

This article has been downloaded from IOPscience. Please scroll down to see the full text article.

2010 J. Phys. A: Math. Theor. 43492002

(http://iopscience.iop.org/1751-8121/43/49/492002)

View the table of contents for this issue, or go to the journal homepage for more

Download details:

IP Address: 132.199.97.29

The article was downloaded on 25/05/2011 at 18:07

Please note that terms and conditions apply. 
FAST TRACK COMMUNICATION

\title{
Unexpected systematic degeneracy in a system of two coupled Gaudin models with homogeneous couplings
}

\author{
B Erbe and J Schliemann \\ Department of Physics, University of Regensburg, 93040 Regensburg, Germany \\ E-mail: Bjoern.Erbe@physik.uni-regensburg.de
}

Received 13 September 2010

Published 18 November 2010

Online at stacks.iop.org/JPhysA/43/492002

\begin{abstract}
We report an unexpected systematic degeneracy between different multiplets in an inversion symmetric system of two coupled Gaudin models with homogeneous couplings, as occurring for example in the context of solid state quantum information processing. We construct the full degenerate subspace (being of macroscopic dimension), which turns out to lie in the kernel of the commutator between the two Gaudin models and the coupling term. Finally we investigate to what extent the degeneracy is related to the inversion symmetry of the system and find that indeed there is a large class of systems showing the same type of degeneracy.
\end{abstract}

PACS numbers: $03.65 .-\mathrm{w}, 76.20 .+\mathrm{q}$, 76.60.Es, 85.35.Be

\section{Introduction}

In a large variety of nanostructures, spins couple to a bath of other spin degrees of freedom. Commonly such systems are described by so-called central spin models. Important examples are given by semiconductor [1-4] and carbon nanotube [5] quantum dots, phosphorus donors in silicon [6], nitrogen vacancy centers in diamond [7-9] and molecular magnets [10]. Motivated by the potential to utilize the central spins as qubits $[11,12]$ or to effectively access the bath spins via the central spins $[13,14]$, central spin models are currently the subject of extensive theoretical and experimental research. However, their importance in a more mathematical context already became clear several decades ago, when Gaudin proved the Bethe ansatz integrability of the central spin model with one central spin (Gaudin model) [15]. Since then they are the focus of the important field of quantum integrability [16-21].

It is well known that the energy levels of a quantum system usually tend to repel each other and degeneracies are exceptional events [22]. Hence, there are only extremely few examples of systems with degenerate eigenstates and even fewer whose eigenstates are systematically degenerate. Famous examples are given by the hydrogen atom [23], the $n$-dimensional harmonic oscillator [24] or the Haldane-Shastry model [25, 26]. In all three cases the degeneracies are due to hidden symmetries requiring a dedicated analysis. 
In this work, we study the spectrum of an inversion symmetric central spin model consisting of two coupled Gaudin models with homogeneous coupling constants, meaning they are chosen to be equal to each other. In order to lower the dimension of the problem, the baths of the two Gaudin models are approximated by single long spins, which do not change the set of eigenvalues of the Hamiltonian. Surprisingly, the resulting Hamiltonian exhibits systematically degenerate multiplets of consecutive total angular momentum and alternating parity, a situation somewhat similar to the degenerate multiplets of orbital angular momentum in the hydrogen atom.

The outline of the paper is as follows. The degeneracies in the coupled Gaudin models are first analyzed in a numerical approach in section 2 . In section 3 we analytically construct the full subspace of degenerate states which turns out to be located in the kernel of the commutator between the two Gaudin models and the coupling term. In section 4 we investigate the role of the inversion symmetry and show that indeed there is a whole class of systems with spectra showing the same type of degeneracy.

\section{Model and spectral properties}

The Gaudin model [15] describes the coupling of a central spin $\vec{S}_{i}$ to a set of $n_{i}$ bath spins $\vec{I}_{i}^{j}$

$$
H_{i}=\vec{S}_{i} \sum_{j=1}^{n_{i}} A_{i}^{j} \vec{I}_{i}^{j}
$$

via some coupling constants $A_{i}^{j}$, which have the unit of energy. In the following we choose the couplings to be homogeneous, i.e. $A_{i}^{j}=A_{i}$. In this case the central spin couples to a simple sum of spins, denoted by $\vec{I}_{i}$ from now on. Furthermore we assume $S_{i}=1 / 2$. Coupling together two such Gaudin models $H_{G}:=H_{1}+H_{2}$ by

$$
H_{c}=J_{\mathrm{ex}} \vec{S}_{1} \vec{S}_{2}
$$

finally yields the Hamiltonian subject to our investigation:

$$
H=H_{G}+H_{c}=A_{1} \vec{S}_{1} \vec{I}_{1}+A_{2} \vec{S}_{2} \vec{I}_{2}+J_{\mathrm{ex}} \vec{S}_{1} \vec{S}_{2} .
$$

The parameter $J_{\text {ex }}$ can be viewed as an exchange coupling. Obviously the Hamiltonian conserves the total spin $\vec{J}=\vec{S}_{1}+\vec{S}_{2}+\vec{I}_{1}+\vec{I}_{2}$ as well as $\vec{I}_{1}^{2}$ and $\vec{I}_{2}^{2}$.

The $n_{i}$ bath spins couple to different values $I_{i}$ of the total bath spin squared. In the following we study the spectrum of the Hamiltonian for $A_{1}=A_{2}=A$, where $A:=(1 / 2)\left(A_{1}+A_{2}\right)$, on subspaces $I_{1}=I_{2}=: I$. On these subspaces, in addition to the symmetries mentioned above, $H$ is invariant under 'inversions', meaning an interchange $1 \leftrightarrow 2$. It is clear that this is not the case globally, i.e. on the entire Hilbert space. However, subspaces with $I_{1}=I_{2}$ lie fully in the kernel of the commutator $[H, \tau]$, where $\tau$ denotes the inversion operator. Obviously this only has the two eigenvalues $( \pm 1)$. In the following we refer to this as positive and negative parity.

In order to reduce the dimension of the problem, we approximate each bath by one single spin of length $I$. This neglects the quantum numbers associated with a certain Clebsch-Gordan decomposition of the respective bath and therefore the multiplicity of the eigenvalues changes, but not the set of eigenvalues itself. Every energy in the resulting spectrum indeed appears $x_{1} x_{2}$ times in the spectrum of $H$, where $x_{i}$ denotes the number of multiplets with the quantum number $I_{i}$. If for example $I_{i}^{j}=\frac{1}{2}$, we have [27]

$$
x_{i}=\left[\left(\begin{array}{c}
n_{i} \\
\frac{n_{i}}{2}-I_{i}
\end{array}\right)-\left(\begin{array}{c}
n_{i} \\
\frac{n_{i}}{2}-I_{i}-1
\end{array}\right)\right] .
$$




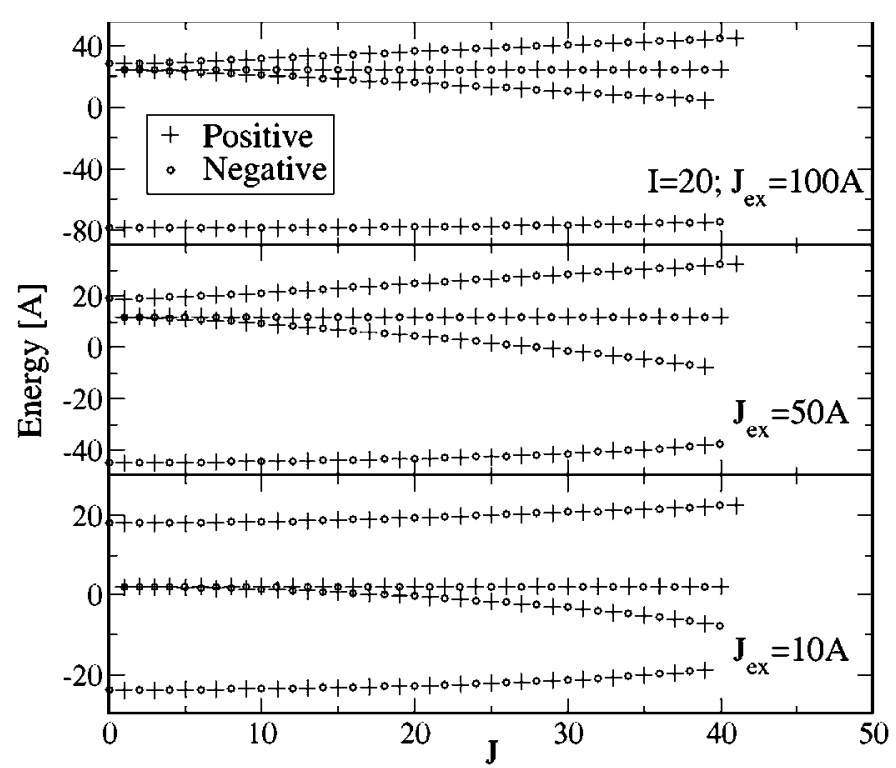

Figure 1. Spectrum of $H$ with baths approximated by two single spins of equal length $I=20$ for different values of the exchange coupling $J_{\text {ex }}$. The energies (in units of $A$ ) are plotted against the total spin $J$, i.e. each data point represents a multiplet of $2 J+1$ states. States of positive (negative) parity are signaled by a cross (circle). For all exchange couplings $J_{\mathrm{ex}}$ we have four 'branches' of energy levels, where the above three originate from triplet states with respect to $H_{c}$ and the lower one is associated with the singlet state. The triplet branch of intermediate energy consists completely degenerate multiplets of alternating parity.

However, it should be stressed again that the energy eigenvalues themselves remain unaltered.

In figures 1 and 2 we show spectra obtained numerically for different values of the exchange coupling constant $J_{\mathrm{ex}}$, both for an even and an odd $I$. Although the spectra are quite rich in detail, their global structure becomes already plausible from simple qualitative arguments. Obviously we always have four 'branches' of energy levels, where, in particular for large $J_{\mathrm{ex}}$, three of them form a bundle separated from the fourth. The three former branches consist of states where the two central spins are predominantly coupled to a triplet (which has eigenvalue $J_{\mathrm{ex}} / 4$ under $H_{c}$ ) while in the latter branch the central spins are mainly in the singlet state (having eigenvalue $-3 J_{\mathrm{ex}} / 4$ under $H_{c}$ ). The coupling of the central spin triplet and singlet to the bath spins then leads to the observed further energy splittings between and within the corresponding branches.

An unexpected particular feature, however, occurs in the triplet branch of intermediate energy. Here all multiplets are energetically completely degenerate with eigenvalue $\left(J_{\text {ex }}-2 A\right) / 4$. These multiplets have consecutive total spin between $J=1$ and $J=2 I$ and alternating parity. Here positive (negative) parity corresponds to $2 I-J$ being even (odd). The latter observation is reminiscent of the degenerate multiplets of orbital angular momentum found in the hydrogen problem. In general, such systematic degeneracies are extremely rare, and hence our finding is interesting in its own right. Moreover, since the degenerate subspace is of particularly high dimension, potential applications in, for example, solid state quantum information processing can be envisaged. It is clear that states with overlap exclusively in a degenerate subspace do not show any non-trivial time evolution. Therefore, 


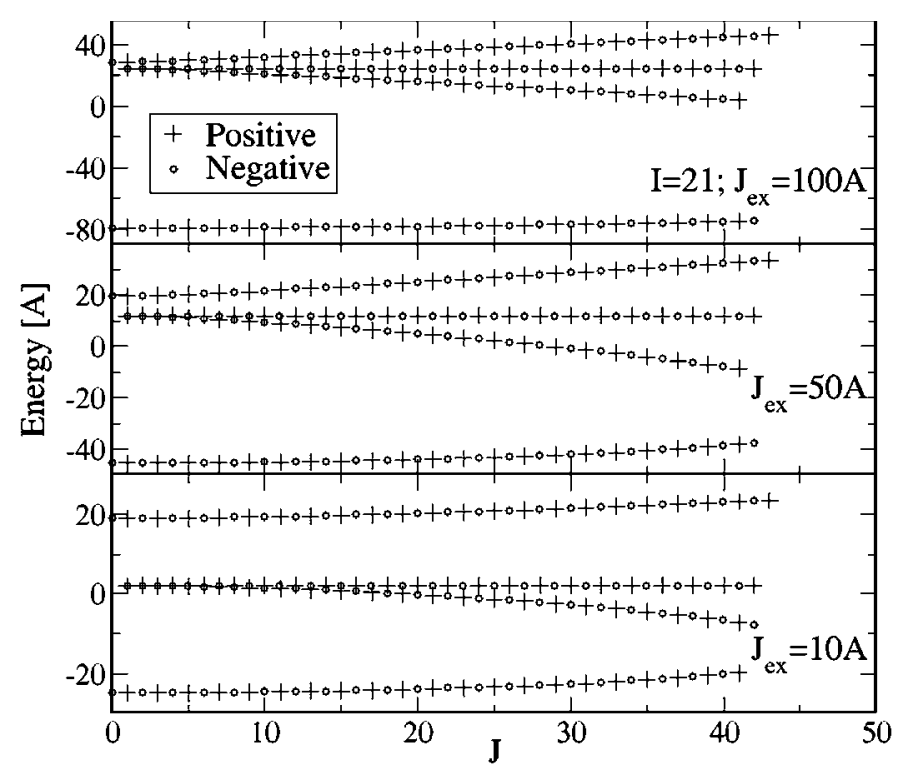

Figure 2. Analogous data as in figure 1 for an odd spin length $I=21$. Again we find a completely degenerate triplet branch.

such spaces have the potential to provide valuable implementations of long lived quantum memory, where the present one appears to be particularly suitable due to its enormous size. Note that even in the 'thermodynamic limit' $I \gg 1$ a fourth of the Hilbert space is degenerate. The dimension of the full Hilbert space is $4(2 I+1)^{2}$ and the degenerate subspace $\mathcal{H}_{D}$ has dimension $\sum_{n=1}^{2 I}(2 n+1)=4 I(I+1)$, yielding

$$
\frac{I(I+1)}{(2 I+1)^{2}} \approx \frac{1}{4},
$$

if $I \gg 1$. Furthermore, the space of degenerate states detected here decomposes into subspaces of different parity which could also serve as a computational basis for quantum information processing.

\section{Construction of the degenerate subspace}

So far we have reported on numerical observations revealing an unexpected systematic degeneracy in the spectrum. In the following we analytically construct the subspace $\mathcal{H}_{D}$ of these degenerate multiplets.

\subsection{General ansatz and first consequences}

As we see below, the degenerate states are simultaneous eigenstates of the Gaudin part $H_{G}$ of the Hamiltonian and the coupling $H_{c}$ between the central spins. In other words, $\mathcal{H}_{D}$ lies entirely in the kernel of the commutator

$$
\left[H_{G}, H_{c}\right]=-\mathrm{i} A J_{\mathrm{ex}}\left(\vec{S}_{1} \times \vec{S}_{2}\right) \cdot\left(\vec{I}_{1}-\vec{I}_{2}\right) .
$$


Let us first turn to a single Gaudin Hamiltonian, $H_{i}=A \vec{S}_{i} \vec{I}_{i}$, with $S_{i}=1 / 2$ and $I_{i}=I$. The eigenvalues read

$$
E_{ \pm}(A, I)=\frac{A}{2}\left( \pm\left(I+\frac{1}{2}\right)+\frac{1}{2}\right)
$$

and the eigenstates are given by a well-known Clebsch-Gordan decomposition [28]

$$
\left|I \pm \frac{1}{2}, m_{i}\right\rangle=\mu^{ \pm}\left(m_{i}\right)|\uparrow\rangle\left|I, m_{i}-\frac{1}{2}\right\rangle \pm \mu^{\mp}\left(m_{i}\right)|\downarrow\rangle\left|I, m_{i}+\frac{1}{2}\right\rangle,
$$

where, apart from standard notation, we have introduced

$$
\mu^{ \pm}(m)=\sqrt{\frac{I \pm m+\frac{1}{2}}{2 I+1}} .
$$

The eigenvalues of $H_{G}=H_{1}+H_{2}$ now follow immediately

$$
\begin{aligned}
& H_{G}\left|+, m_{1}\right\rangle\left|+, m_{2}\right\rangle=\frac{A}{2}\left(I_{1}+I_{2}\right)\left|+, m_{1}\right\rangle\left|+, m_{2}\right\rangle \\
& H_{G}\left|+, m_{1}\right\rangle\left|-, m_{2}\right\rangle=-\frac{A}{2}\left|+, m_{1}\right\rangle\left|-, m_{2}\right\rangle \\
& H_{G}\left|-, m_{1}\right\rangle\left|+, m_{2}\right\rangle=-\frac{A}{2}\left|-, m_{1}\right\rangle\left|+, m_{2}\right\rangle \\
& H_{G}\left|-, m_{1}\right\rangle\left|-, m_{2}\right\rangle=-\frac{A}{2}\left(I_{1}+I_{2}+2\right)\left|-, m_{1}\right\rangle\left|-, m_{2}\right\rangle,
\end{aligned}
$$

where we abbreviated $\left|I \pm \frac{1}{2}, m_{i}\right\rangle=:\left| \pm, m_{i}\right\rangle$. Obviously, the states $\left| \pm, m_{1}\right\rangle\left|\mp, m_{2}\right\rangle$ are degenerate with the eigenvalue being independent of $I$. As seen above, the highly degenerate eigenvalue in the subspace $\mathcal{H}_{D}$ is $\left(J_{\mathrm{ex}}-2 A\right) / 4$. Thus, eigenstates of $H$ with this eigenvalue can be constructed by simply combining the states $\left| \pm, m_{1}\right\rangle\left|\mp, m_{2}\right\rangle$ to triplet states with respect to the two central spins, meaning that they lie in the kernel of (5). At this point it is of course not clear that all eigenstates with the above eigenvalue are resulting through this approach. However, we will see that this is indeed the case.

In other words, our goal is to eliminate singlet contributions from suitable linear combinations of the states $\left| \pm, m_{1}\right\rangle\left|\mp, m_{2}\right\rangle$. To this end we use an ansatz already accounting for the conservation of $J^{z}$ and the parity symmetry by superimposing states of the form

$$
|+, m\rangle|-, M-m\rangle \pm|-, M-m\rangle|+, m\rangle
$$

where $M$ is the eigenvalue of $J^{z}$. All considerations will focus on $M \geqslant 0$, as states with $M<0$ result simply by reversing every spin. In the following analysis one needs to distinguish the four different cases depending on whether $M$ is even or odd, and $I$ is integer or half-integer. This case-by-case procedure can be nicely encapsulated and simplified as follows by introducing $i=2 I-M$ with $i=0, \ldots, 2 I$. In figure 3 the possible values of $m_{1}$ and $m_{2}$ are arranged on a grid. The diagonal lines mark the states of constant magnetization $M=2 I-i$, where we refer to the maximal value on such a diagonal as $m_{\max }$. Obviously, we have $m_{\max }=I-1 / 2$ for $i=2 I$ and $m_{\max }=I+1 / 2$ otherwise. Following a line of constant magnetization starting from $m_{\max }$, one recognizes that from a certain value $m=m_{\min }$ on, all occurring states result from those with larger values of $m$ by interchanging the respective magnetizations $(m, M-m)$. In figure 3 these 'complementary' states are connected by dotted bended lines. It is easy to see that if $i$ is odd, we have $m_{\min }=(2 I-i) / 2$, whereas for an even value of $i$ we have to add $(1 / 2)$ so that $m_{\min }=(2 I-i+1) / 2$. It is now a simple fact that there are states which do not have a complement. This is the case for the states with $m=m_{\max }$ if $i \neq 2 I$ and for those with $m=m_{\min }$ provided $i$ is odd or equal to zero. 


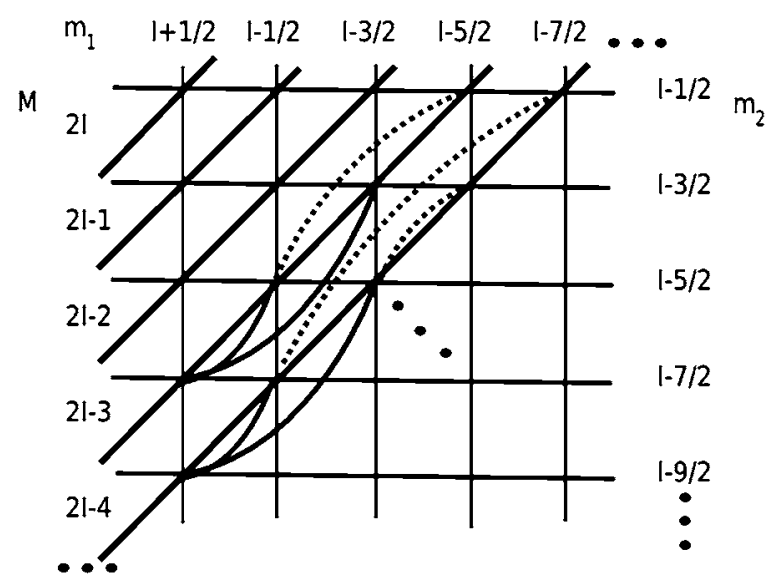

Figure 3. The different values of $m_{1}$ and $m_{2}$ arranged on a grid. The diagonal lines mark the states with constant magnetization. The dotted curved lines connect the states with interchanged magnetization, combined in our ansatz (11). The solid curved lines connect the states which are combined in order to construct the set of linearly independent eigenstates spanning the eigenspace to the eigenvalue $(J-2 A) / 4$.

With respect to later considerations it turns out to be more convenient to use an ansatz which is a sum over pairs of complementary states, rather than a direct superposition of the states (10). Hence we introduce coefficients $\alpha_{m}, \alpha_{m}^{\prime}$ for any state with $m \geqslant m_{\min }$ and its complement and combine them to a sum running from $m_{\min }$ to $m_{\max }$ :

$$
\begin{aligned}
| \pm, i\rangle:= & \sum_{m=m_{\min }}^{m_{\max }}\left[\alpha_{m}(|+, m\rangle|-, 2 I-i-m\rangle \pm|-, 2 I-i-m\rangle|+, m\rangle)\right. \\
& \left.\quad+\theta(m) \alpha_{m}^{\prime}(|+, 2 I-i-m\rangle|-, m\rangle \pm|-, m\rangle|+, 2 I-i-m\rangle)\right]
\end{aligned}
$$

The complements of the respective $m=m_{\max }$ states automatically vanish, whereas the function $\theta(m)$ accounts for the $m_{\min }$ states without a complement:

$$
\theta(m)=\Theta\left(m-m_{\min }-1\right)+\delta_{i \bmod 2,0} \delta_{m, m_{\min }}
$$

where the Heavyside function $\Theta(x)$ is unity for any $x \geqslant 0$ and zero otherwise.

Clearly, ansatz (11) is an eigenstate of $H_{G}$,

$$
H_{G}| \pm, i\rangle=-\frac{A}{2}| \pm, i\rangle
$$

consisting of triplet and singlet terms. Eliminating the latter by demanding

$$
\begin{aligned}
\sum_{m=m_{\min }}^{m_{\max }}\left[\left(\alpha_{m} \mu^{+}(m) \mu^{+}(2 I-i-m) \mp \theta(m) \alpha_{m}^{\prime} \mu^{-}(m) \mu^{-}(2 I-i-m)\right)\right. \\
\quad \times\left(\left|m-\frac{1}{2}, 2 I-i-m+\frac{1}{2}\right\rangle \mp\left|2 I-i-m+\frac{1}{2}, m-\frac{1}{2}\right\rangle\right) \\
+\left(\alpha_{m} \mu^{-}(m) \mu^{-}(2 I-i-m) \mp \theta(m) \alpha_{m}^{\prime} \mu^{+}(m) \mu^{+}(2 I-i-m)\right) \\
\left.\times\left(\left|m+\frac{1}{2}, 2 I-i-m-\frac{1}{2}\right\rangle \mp\left|2 I-i-m-\frac{1}{2}, m+\frac{1}{2}\right\rangle\right)\right]=0,
\end{aligned}
$$

we arrive at an eigenstate of $H_{c}$. 
Let us first consider the two particularly simple cases $i=0$ and $i=2 I$. For $i=0$, i.e. $M=2 I$, the sum consists of only one term $m=I+1 / 2$. In this case the contributions related $\alpha_{I+1 / 2}^{\prime}$ to in (14a) and (14b) are vanishing, implying that for positive parity the unwanted singlet terms are automatically zero. This means that the largest degenerate multiplet with $J=2 I$ always has positive parity, as demonstrated by our numerics.

In the other case $i=2 I$, i.e. $M=0$; one easily sees that

$$
\mu^{+}(m) \mu^{+}(-m)=\mu^{-}(m) \mu^{-}(-m) .
$$

If $i$ is even, this condition means that for every $m$ the singlet terms can be eliminated by simply choosing $\alpha_{m}= \pm \alpha_{m}^{\prime}$. Therefore, in this case we always have an equal number of multiplets with positive and with negative parity. As mentioned above, for an odd value of $i$ the summand with $m=m_{\min }=0$ does not have a complement. However, for positive parity the unwanted terms vanish automatically so that the number of positive multiplets is larger by 1 than the number of negative multiplets. In total, we get $2 I$ solutions as suggested by our numerics.

These solutions result from demanding that the terms in (14a) and (14b) vanish separately, while, strictly speaking, only their sum is required to be zero. However, it is indeed simple to see that there are no further solutions. Demanding that the total sum vanishes leads to the conditions

$$
\mu^{+}(m) \mu^{+}(-m)\left(\alpha_{m} \mp \alpha_{m}^{\prime}\right)= \pm \mu^{+}(m-1) \mu^{+}(-m+1)\left(\alpha_{m-1} \mp \alpha_{m-1}^{\prime}\right),
$$

and $\left(\alpha_{\frac{1}{2}} \mp \alpha_{\frac{1}{2}}^{\prime}\right)=\left(\alpha_{I-\frac{1}{2}} \mp \alpha_{I-\frac{1}{2}}^{\prime}\right)=0$, which obviously give the same solutions as above. In summary, the resulting eigenstates at $i=2 I(M=0)$ can be formulated most compactly as

$$
\begin{aligned}
& | \pm, 2 I, m\rangle:=|\uparrow \uparrow\rangle\left(\left|m-\frac{1}{2},-m-\frac{1}{2}\right\rangle \pm\left|-m-\frac{1}{2}, m-\frac{1}{2}\right\rangle\right) \\
& -|\downarrow \downarrow\rangle\left(\left|m+\frac{1}{2},-m+\frac{1}{2}\right\rangle \pm\left|-m+\frac{1}{2}, m+\frac{1}{2}\right\rangle\right) .
\end{aligned}
$$

That $\mathcal{H}_{D}$ lies fully in the kernel of (5) becomes clear at this point. There are $2 I$ degenerate multiplets with alternating parity, each of which gives one state with $M=0$. Above we constructed states which are superpositions of exactly those states and lie in the kernel of (5). They can be combined to give eigenstates of $\vec{J}^{2}$, so that $\mathcal{H}_{D}$ can be constructed simply by applying $J^{ \pm}$. From $\left[\vec{J}, H_{G}\right]=\left[\vec{J}, H_{c}\right]=0$ it follows that

$$
\left[J^{ \pm},\left[H_{G}, H_{c}\right]\right]=0,
$$

meaning that a state resulting from the application of $J^{ \pm}$to a state lying in the kernel of (5) again lies in the kernel of (5). Therefore the full degenerate subspace is located there.

\subsection{Complete construction}

Now we come to the construction of the full degenerate space $\mathcal{H}_{D}$. In an immediate approach we follow the route described above and combine the states (16) to eigenstates of $\vec{J}^{2}$ such that $\mathcal{H}_{D}$ can be generated by applying $J^{ \pm}$. Unfortunately the construction of $\vec{J}^{2}$ eigenstates is possible only up the solution of a homogeneous set of equations with a (symmetric) tridiagonal coefficient matrix, which has to be carried out numerically. Due to the simple shape of the matrix such a problem has the very low complexity of $\mathcal{O}(2 I)$ so that even systems of realistic size with respect to experimental situations in for example semiconductor quantum dots $I \sim 10^{6}$ can be treated on conventional computers [1-4]. However, in a second approach we construct a basis of $\mathcal{H}_{D}$ in a fully analytical fashion. The resulting basis states are eigenstates 
of $J^{z}$ and $\tau$, but they are neither orthogonal nor do they satisfy the $\vec{J}^{2}$ symmetry. Nevertheless, for both applied as well as more mathematical future considerations it will be helpful to have closed analytical expressions at hand.

3.2.1. First approach: construction of eigenstates of $\vec{J}^{2}$ with $M=0 . \quad$ As mentioned above, our first approach consists in using the particularly simple solutions for $i=2 I$ given in (16) by combining them to eigenstates of $\vec{J}^{2}$ such that applying the ladder operators $J^{ \pm}$generates the full space $\mathcal{H}_{D}$. Hence we demand

$$
\begin{aligned}
\vec{J}^{2} \sum_{m=m_{\min }}^{I-\frac{1}{2}} \beta_{m}| \pm, 2 I, m\rangle & =J(J+1) \sum_{m=m_{\min }}^{I-\frac{1}{2}} \beta_{m}| \pm, 2 I, m\rangle \\
& \Leftrightarrow \vec{J}^{2} \sum_{m=m_{\min }}^{I-\frac{1}{2}} \beta_{m}| \pm, 2 I, m\rangle-J(J+1) \sum_{m=m_{\min }}^{I-\frac{1}{2}} \beta_{m}| \pm, 2 I, m\rangle=0 .
\end{aligned}
$$

Explicitly this reads

$$
\begin{aligned}
|\uparrow \uparrow\rangle \sum_{m=m_{\min }}^{I-\frac{1}{2}} \beta_{m} & {\left[\left(2 I(I+1)-2\left(m+\frac{1}{2}\right)\left(m-\frac{1}{2}\right)-J(J+1)\right)\right.} \\
& \times\left(\left|m-\frac{1}{2},-m-\frac{1}{2}\right\rangle \pm\left|-m-\frac{1}{2}, m-\frac{1}{2}\right\rangle\right) \\
& +v^{+}\left(m-\frac{1}{2}\right) v^{-}\left(-m-\frac{1}{2}\right)\left(\left|m+\frac{1}{2},-m-\frac{3}{2}\right\rangle \pm\left|-m-\frac{3}{2}, m+\frac{1}{2}\right\rangle\right) \\
& \left.+v^{-}\left(m-\frac{1}{2}\right) v^{+}\left(-m-\frac{1}{2}\right)\left(\left|m-\frac{3}{2},-m+\frac{1}{2}\right\rangle \pm\left|-m+\frac{1}{2}, m-\frac{3}{2}\right\rangle\right)\right] \\
& -|\downarrow \downarrow\rangle \sum_{m=m_{\min }} \beta_{m}\left[\left(2 I(I+1)-2\left(m+\frac{1}{2}\right)\left(m-\frac{1}{2}\right)-J\left(J+\frac{1}{2}\right)\right)\right. \\
& \times\left(\left|m+\frac{1}{2},-m+\frac{1}{2}\right\rangle \pm\left|-m+\frac{1}{2}, m+\frac{1}{2}\right\rangle\right) \\
& +v^{+}\left(m+\frac{1}{2}\right) v^{-}\left(-m+\frac{1}{2}\right)\left(\left|m+\frac{3}{2},-m-\frac{1}{2}\right\rangle \pm\left|-m-\frac{1}{2}, m+\frac{3}{2}\right\rangle\right) \\
& \left.+v^{-}\left(m+\frac{1}{2}\right) v^{+}\left(-m-\frac{1}{2}\right)\left(\left|m-\frac{1}{2},-m+\frac{3}{2}\right\rangle \pm\left|-m+\frac{3}{2}, m-\frac{1}{2}\right\rangle\right)\right]=0,
\end{aligned}
$$

where $v^{ \pm}(m)=\sqrt{I(I+1)-m(m \pm 1)}$ and hence

$$
\begin{aligned}
& v^{+}\left(m-\frac{1}{2}\right) v^{-}\left(-m-\frac{1}{2}\right)=v^{+}\left(m+\frac{1}{2}\right) v^{-}\left(-m+\frac{1}{2}\right) \\
& v^{-}\left(m-\frac{1}{2}\right) v^{+}\left(-m-\frac{1}{2}\right)=v^{-}\left(m+\frac{1}{2}\right) v^{+}\left(-m-\frac{1}{2}\right),
\end{aligned}
$$

which is plausible, because the $|\uparrow \uparrow\rangle$ and $|\downarrow \downarrow\rangle$ terms must vanish separately. Note that all components with $|\uparrow \downarrow\rangle,|\downarrow \uparrow\rangle$ are equal to zero. It is now simple to see that the state in (18b) 
for some $m$ is identical to the one in $(18 b)$ for $(m+1)$ and to the one in $(18 c)$ for $(m+2)$. For an even value of $i$ eliminating these terms gives the following set of equations:

$$
\begin{gathered}
\beta_{m}\left[v^{+}\left(m-\frac{1}{2}\right) v^{-}\left(-m-\frac{1}{2}\right)\right]+\beta_{m+1}\left[2 I(I+1)-2\left(m+\frac{3}{2}\right)\left(m+\frac{1}{2}\right)-J(J+1)\right] \\
+\Theta\left(I-\frac{3}{2}-m\right) \beta_{m+2}\left[v^{+}\left(m+\frac{3}{2}\right) v^{-}\left(-m-\frac{5}{2}\right)\right]=0 \\
\beta_{\frac{1}{2}}[2 I(I+1)-J(J+1) \pm I(I+1)]+\beta_{\frac{3}{2}}\left[v^{-}(1) v^{+}(-2)\right]=0,
\end{gathered}
$$

where $m=1 / 2, \ldots, I-3 / 2$. This yields a symmetric tridiagonal matrix. However, the symmetry of the matrix is destroyed if $i$ is odd. In this case we have

$$
\begin{gathered}
\beta_{m}\left(1 \pm \delta_{m, 0}\right)\left[v^{+}\left(m-\frac{1}{2}\right) v^{-}\left(-m-\frac{1}{2}\right)\right]+\beta_{m+1}\left[2 I(I+1)-2\left(m+\frac{3}{2}\right)\left(m+\frac{1}{2}\right)-J(J+1)\right] \\
+\Theta\left(I-\frac{3}{2}-m\right) \beta_{m+2}\left[v^{+}\left(m+\frac{3}{2}\right) v^{-}\left(-m-\frac{5}{2}\right)\right]=0 \\
\beta_{0}\left[2 I(I+1)-J(J+1)-\frac{1}{2}\right]+\beta_{1}\left[v^{-}\left(\frac{1}{2}\right) v^{+}\left(-\frac{3}{2}\right)\right]=0,
\end{gathered}
$$

where $m=0, \ldots, I-1 / 2$. The two above systems now have to be solved numerically for the different values of $J$.

3.2.2. Second approach: explicit elimination of singlet contributions via ansatz. Our second approach, which in contrast to the one above will lead to closed analytical expressions for the degenerate eigenstates, consists in directly determining the constants $\alpha_{m}, \alpha_{m}^{\prime}$ for every value of $M$. As already used above, considering (14a) for some $m$ and $(14 b)$ for $(m-1)$, one sees that the respective states become identical up to a factor $(\mp 1)$. The idea is now to eliminate these terms systematically, so that we get a sufficient number of linearly independent eigenvectors. As indicated in figure 3 by the solid curved lines, this can be done by simply superposing an increasing number of successive terms and choosing all other constants to be equal to zero. Of course these solutions are by no means unique; we just choose the most compact ones. For an odd value of $i$ this yields the following quite cumbersome solutions:

$$
\begin{gathered}
\alpha_{I+\frac{1}{2}-\lambda}=(-1)^{\kappa-\lambda}(\mp 1)^{\kappa-\lambda-1} N_{\kappa}\left[\mu^{+}\left(I+\frac{1}{2}-\lambda\right) \mu^{+}\left(I-i-\frac{1}{2}+\lambda\right)\right. \\
\left.\mp \mu^{-}\left(I+\frac{1}{2}-\lambda\right) \mu^{-}\left(I-i-\frac{1}{2}+\lambda\right)\right]^{-1},
\end{gathered}
$$

where $\lambda=0, \ldots,(\kappa-1)$ and

$$
=\left\{\begin{array}{l}
N_{\kappa} \\
{\left[\mu^{-}\left(I+\frac{1}{2}-\kappa\right) \mu^{-}\left(I-i-\frac{1}{2}+\kappa\right)-\frac{\left(\mu^{+}\left(I+\frac{1}{2}-\kappa\right) \mu^{+}\left(I-i-\frac{1}{2}+\kappa\right)\right)^{2}}{\mu^{-}\left(I+\frac{1}{2}-\kappa\right) \mu^{-}\left(I-i-\frac{1}{2}+\kappa\right)}\right] \alpha_{I+\frac{1}{2}-\kappa}} \\
{\left[\left(\mu^{+}\left(I-\frac{i}{2}\right)\right)^{2} \mp\left(\mu^{-}\left(I-\frac{i}{2}\right)\right)^{2}\right] \alpha_{I-\frac{i}{2}},}
\end{array}\right.
$$

where the first line refers to $\kappa=1, \ldots,(i-1) / 2$ and the second line applies to $\kappa=(i+1) / 2$. For even $i$ and negative parity the solution coincides with the first line of (3.2.2), where now $\kappa=1, \ldots, i / 2$. Considering positive parity we get

$N_{\kappa}$

$$
=\left\{\begin{array}{l}
{\left[\mu^{-}\left(I+\frac{1}{2}-\kappa\right) \mu^{-}\left(I-i-\frac{1}{2}+\kappa\right)-\frac{\left(\mu^{+}\left(I+\frac{1}{2}-\kappa\right) \mu^{+}\left(I-i-\frac{1}{2}+\kappa\right)\right)^{2}}{\mu^{-}\left(I+\frac{1}{2}-\kappa\right) \mu^{-}\left(I-i-\frac{1}{2}+\kappa\right)}\right] \alpha_{I+\frac{1}{2}-\kappa}} \\
{\left[\mu^{+}\left(I-\frac{i}{2}+\frac{1}{2}\right) \mu^{+}\left(I-\frac{i}{2}-\frac{1}{2}\right)-\mu^{-}\left(I-\frac{i}{2}+\frac{1}{2}\right) \mu^{-}\left(I-\frac{i}{2}-\frac{1}{2}\right)\right] \alpha_{I-\frac{i}{2}+\frac{1}{2}}}
\end{array}\right.
$$

with $\kappa=1, \ldots,(i-2) / 2$ for the first line and $\kappa=i / 2$ for the second one. Due to the presence of an $\alpha_{m_{\min }}^{\prime}$ term, in contrast to the case of an odd $i$, here we have an additional solution. This 
Table 1. Numerically detected degeneracy pattern.

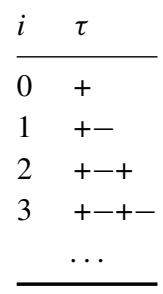

results by simply choosing all constants to be equal to zero except for $\alpha_{m_{\min }}$ and $\alpha_{m_{\min }}^{\prime}$, which are determined by eliminating the $(14 b)$ term:

$$
\alpha_{I-\frac{i}{2}+\frac{1}{2}}^{\prime}=\mp \frac{\mu^{-}\left(I-\frac{i}{2}+\frac{1}{2}\right) \mu^{-}\left(I-\frac{i}{2}-\frac{1}{2}\right)}{\mu^{+}\left(I-\frac{i}{2}+\frac{1}{2}\right) \mu^{+}\left(I-\frac{i}{2}-\frac{1}{2}\right)} \alpha_{I-\frac{i}{2}+\frac{1}{2}} .
$$

Note that all the remaining constants are determined by the normalization condition. Let us give a brief discussion of the above results. With respect to subspaces of fixed $i$, the degeneracies shown in figures 1 and 2 yield the pattern shown in table 1. Obviously for any $i$ there are $(i+1)$ states. If $i$ is odd, there is an equal number of states with positive and with negative parity, whereas for an even value of $i$ the number of states with positive parity is larger by one than the number of states with negative parity. This is perfectly reproduced by our solutions. For an odd $i$ the index $\kappa$ in (3.2.2) runs up to $(i+1) / 2$ for each parity, meaning that there are $(i+1)$ solutions in total. If $i$ is even, (3.2.2) yields $i / 2$ solutions for each parity and an additional one for positive parity.

\section{The role of the inversion symmetry}

In the preceding section we constructed the full degenerate subspace by determining the coefficients in (11) so that we arrive at triplet states of the two central spins. Obviously, such a construction is still possible if inversion symmetry is broken. Note that if $I_{1} \neq I_{2}$, additional labels for the spin length have to be introduced in (8) and (9). However, it is simple to see that in general our states are no longer eigenstates with respect to $H_{G}$, because the degeneracy between the $H_{G}$ eigenstates $\left| \pm, m_{1}\right\rangle\left|\mp, m_{2}\right\rangle$ is lifted. Indeed this can be easily recovered by demanding $E_{+}\left(A_{1}, I_{1}\right)+E_{-}\left(A_{2}, I_{2}\right)=E_{-}\left(A_{1}, I_{1}\right)+E_{+}\left(A_{2}, I_{2}\right)$, yielding the quite remarkable relation

$$
A_{1} \operatorname{dim}\left(\mathcal{H}_{1}\right)=A_{2} \operatorname{dim}\left(\mathcal{H}_{2}\right),
$$

where $\operatorname{dim}\left(\mathcal{H}_{i}\right)=2 I_{i}+1$ denotes the dimension of the Hilbert space associated with $I_{i}$. Note that the bath spins $\sum_{j=1}^{n_{i}} \vec{I}_{i}^{j}$ couple to different values of $I_{i}$ so that, by a corresponding choice of $A_{1}, A_{2}$, several different degenerate subspaces can be constructed.

Relation (21) means that the inversion symmetric case is only an example of a whole class of systems exhibiting the same type of systematic degeneracy. In figure 4 we plot the relevant part of the spectrum for $I_{1} \neq I_{2}$ with $I_{2}>I_{1}$. In the upper panel the couplings violate (21) and consequently the degeneracy between the multiplets is lifted. In the bottom panel it is recovered by choosing $A_{1}, A_{2}$ according to (21), leading to

$$
\begin{aligned}
& A_{1}=\frac{1+2 I_{2}}{I_{1}+I_{2}+1} A \\
& A_{2}=\frac{1+2 I_{1}}{I_{1}+I_{2}+1} A .
\end{aligned}
$$




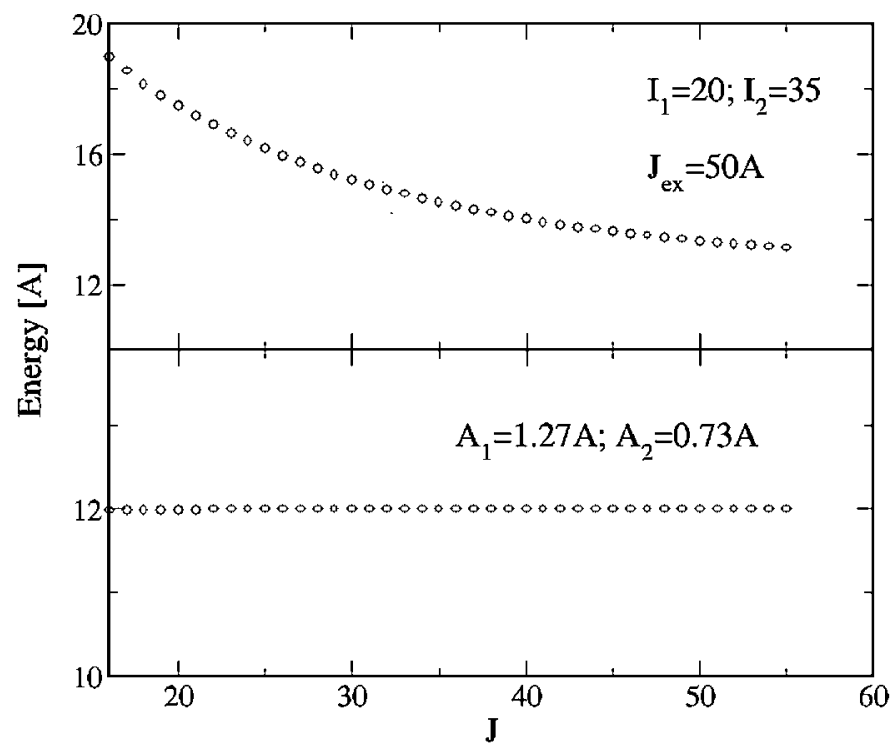

Figure 4. Formerly degenerate branch of the spectrum for $I=20$ and $J=25 A$. The inversion symmetry is broken by choosing $I_{1} \neq I_{2}$. The deviation from the degenerate case is stronger for multiplets with a small value of the quantum number $J$ than for those with a large value. The bottom panel shows the spectrum with the degeneracy recovered by choosing $A_{1} \neq A_{2}$ due to (22).

In direct analogy to the inversion symmetric case the branch begins at $\left(I_{1}+I_{2}\right)$ and ends at $\left(I_{2}-I_{1}+1\right)$.

Relation (21) has a concrete physical meaning. Consider a semiconductor double quantum dot. Here the electron spin interacts with the surrounding nuclear spins via the hyperfine interaction, yielding a system of two coupled Gaudin models. The role of the couplings $A_{1}, A_{2}$ is played by the overall coupling strengths of the respective dots, given the sum of all hyperfine coupling constants (which depend on the properties of the respective material). The size of the Hilbert spaces results from the spatial extent of the respective electron wavefunction. If it is e.g. stretched over a larger area, each individual coupling decreases, but the sum remains unaltered. Hence, in an approximative sense, relation (21) can always be realized by properly adjusting the electron wave function.

With respect to possible future applications of $\mathcal{H}_{D}$ it is important to note that for parameters only weakly violating (21), the multiplets are still nearly degenerate: Let us fix $A_{1}=A_{2}$ and vary $I_{1}, I_{2}$ so that (21) is violated. From the eigenvalues of $H_{G}$ it is clear that the degeneracy is lifted in a continuous way. Furthermore, as can be seen clearly in the upper panel of figure 4 , the influence of $I_{1} \neq I_{2}$ on multiplets with small quantum numbers $J$ is much stronger than on those with large values of $J$. This is also the case if we analogously choose $A_{1} \neq A_{2}$.

\section{Conclusion}

In summary we have reported an unexpected systematic degeneracy in an inversion symmetric system of two coupled Gaudin models with homogeneous couplings. This leads to a degenerate subspace of macroscopic size. We have constructed the complete degenerate subspace, which 
is fully located in the kernel of the commutator between the two Gaudin models and their coupling term. Furthermore we have studied the role of the inversion symmetry. Indeed it turns out that the inversion symmetric case is only an example for a whole family of systems all of which share the same type of systematic degeneracy. This exclusively originates in the degeneracy of two eigenspaces of the Gaudin part of the Hamiltonian, yielding a remarkable relation between the dimension of the bath Hilbert spaces and the couplings.

Nevertheless, so far we have not been able to detect the (possibly continuous) symmetry underlying this remarkable degeneracy, i.e. a set of generating operators that would connect the highly degenerate multiplets. This question remains as an important but probably rather intricate problem for further studies. Furthermore it would be fruitful to study applications of the degenerate space especially in the context of solid state quantum information processing.

\section{Acknowledgment}

This work was supported by Deutsche Forschungsgemeinschaft via SFB631.

\section{References}

[1] Petta J R, Johnson A C, Taylor J M, Laird E A, Yacoby A, Lukin M D, Marcus C M, Hanson M P and Gossard A C 2005 Science 3092180

[2] Koppens F H L, Buizert C, Tielrooij K J, Vink I T, Nowack K C, Meunier T, Kouwenhoven L P and Vandersypen L M K 2006 Nature 442766

[3] Hanson R, Kouwenhoven L P, Petta J R, Tarucha S and Vandersypen L M K 2007 Rev. Mod. Phys. 791217

[4] Braun P-F et al 2005 Phys. Rev. Lett. 94116601

[5] Churchill H, Bestwick A, Harlow J, Kuemmeth F, Marcos D, Stwertka C, Watson S and Marcus C 2009 Nat. Phys. 5321

[6] Abe E, Itoh K M, Isoya J and Yamasaki S 2004 Phys. Rev. B 70033204

[7] Jelezko F, Gaebel T, Popa I, Gruber A and Wrachtrup J 2004 Phys. Rev. Lett. 92076401

[8] Childress L, Dutt M V G, Taylor J M, Zibrov A S, Jelezko F, Wrachtrup J, Hemmer P R and Lukin M D 2006 Science 314281

[9] Hanson R, Dobrovitski V V, Feiguin A E, Gywat O and Awschalom D D 2008 Science 320352

[10] Ardavan A, Rival O, Morton J J L, Blundell S J, Tyryshkin A M, Timco G A and Winpenny R E P 2007 Phys. Rev. Lett. 98057201

[11] Loss D and DiVincenzo D P 1998 Phys. Rev. A 57120

[12] Kane B E 1998 Nature 393133

[13] Schwager H, Cirac J I and Giedke G 2010 Phys. Rev. B 81045309

[14] Schwager H, Cirac J I and Giedke G 2010 New J. Phys. 12043026

[15] Gaudin M 1976 J. Phys. (France) 731087

[16] Garajeu D and Kiss A 2001 J. Math. Phys. 423497

[17] Sklyanin E K and Takebe T 1996 Phys. Lett. A 219217

[18] Frenkel E 2004 arXiv:math0407524v2

[19] Erbe B and Schmidt H-J 2010 J. Phys. A: Math. Theor. 43085215

[20] Schliemann J 2010 Phys. Rev. B 81081301

[21] Erbe B and Schliemann J 2010 Phys. Rev. Lett. 105177602 (arXiv:1006.4983)

[22] von Neumann J and Wigner E P 1929 Phys. Z. 30467

[23] Pauli W 1926 Z. Phys. 36336

[24] Baker G A 1956 Phys. Rev. 1031119

[25] Haldane F D M, Ha Z N C, Talstra J C, Bernard D and Pasquier V 1992 Phys. Rev. Lett. 692021

[26] Frahm H 1993 J. Phys. A: Math. Gen. 26 L473

[27] Schliemann J, Khaetskii A and Loss D 2003 J. Phys.: Condens. Matter 15 R1809

[28] Schwabl F 2002 Quantum Mechanics (Berlin: Springer) chapter 10.3 